\title{
¿País de emigración, inmigración, tránsito y retorno? La formación de un sistema de migración colombiano*
}

Mauricio Palma**

\section{RESUMEN}

A partir de la teoría de los sistemas de migración formulada por Oliver Bakewell (2010, 2013), el artículo argumenta la existencia de un sistema de migración colombiano. Este se estructura con base en la configuración histórica de tres elementos: a) flujos, de personas, ideas y bienes; b) instituciones, en el sentido de discursos y prácticas asociadas; y (c) estrategias, que responden tanto a dinámicas normativas como derivadas del entorno en el que se gesta el fenómeno migratorio contemporáneo. En su configuración se toman en cuenta lógicas económicas y políticas, pero también prácticas culturales, en medio del proceso de transición de Colombia como una sociedad de emigración pura, a ser un país de inmigración, tránsito y retorno.

Palabras clave: migración internacional, sistema de migración colombiano, emigración, inmigración, tránsito, retorno.

\section{A country of emigration, immigration, transit and return? The formation of a Colombian migration system}

\section{ABSTRACT}

Starting from the theory on Migration Systems advanced by Oliver Bakewell (2010, 2013),

\footnotetext{
* El autor agradece a los profesores Adriana Serrano y Andrés Molano-Rojas de la Universidad del Rosario, por sus comentarios e ideas para el desarrollo de este artículo.

** MSc Global History. Profesor de la facultad de Relaciones Internacionales de la Universidad del Rosario, Bogotá (Colombia).mauricio.palma@urosario.edu.co
}

Recibido: 30 de marzo de 2015 / Modificado: 2 de junio de 2015 / Aceptado: 2 de junio de 2015.

Para citar este artículo

Palma, M. (2015). ¿País de emigración, inmigración, tránsito y retorno? La formación de un sistema de migración colombiano. OAsIs, 21, 7-28. DOI: http://dx.doi.org/10.18601/16577558.n21.02 
this article argues for the existence of a $\mathrm{Co}$ lombian Migration System. It is the result of the historical existence of three elements: (a) flows - of people, ideas and goods; (b) institutions - in the sense of discourses and associated practices; and (c) strategies, which respond to normative dynamics, as well as those derived from the environment in which the current migration wave has emerged. Its formation is the result of the interaction between economic and political determinants as well as cultural practices, in the transition of Colombia from a net emigration society into an immigration, transit, and return country.

Key words: International migration, Colombian migration system, emigration, immigration, transit, return.

\section{INTRODUCCIÓN}

La migración humana desde, a través de y hacia Colombia ha sido poco explorada en los estudios internacionales. También es escaso el desarrollo de un cuerpo teórico multidisciplinar para entender las oportunidades y los desafíos que pueden surgir de este fenómeno. Al tiempo, la necesidad de generar políticas públicas al respecto es creciente. Entre los varios intentos que se aproximan al fenómeno se encuentran los aportes desde la historia económica de
Cárdenas y Mejía (2006) y de Mejía-Ochoa (2012), los ejercicios etnográficos de Echeverri (2005), Roll y Gómez (2010), Bermúdez (2011) y Roll (2012), y los enfoques que relacionan los flujos de personas causados por el conflicto armado colombiano y el régimen internacional del asilo y el refugio (Gottwald, 2004; Nagle, 2004).

Sin embargo y pese a la creciente relevancia del tema, son pocos los análisis desde las relaciones internacionales y sus disciplinas afines -como los estudios transnacionales y globales-. Las excepciones son las aproximaciones de Aya et al. (2010), en la que se indaga por la escasa migración hacia Colombia, y de Wagbou et al. (2012), en la que se conceptualiza la tradición de emigración colombiana.

Mientras tanto, en el mundo se ha consolidado un cuerpo teórico para estudiar la migración humana desde las relaciones internacionales. En esta dinámica se han destacado los aportes de Massey et al. (1999), Arango (2000) Wimmer y Glick-Schiller (2003), Castles et al. (2013), entre otros. Dentro de los enfoques multidisciplinares, el aporte de Oliver Bakewell (2013) ha cobrado cierta relevancia recientemente. Este autor ha relanzado y actualizado el concepto de sistema de migración, que había desaparecido del medio académico desde hacía varias décadas ${ }^{1}$. En su aporte logra sintetizar un modelo teórico que sirve, no solo

La idea del enfoques sistémico surgió hacia la década de 1960 con los aportes de Arrighi y Saul, Gutkind y Richards (Bakewell, 2013). Este dejó de ser del interés del mainstream académico ante la postulación de la teoría de redes y otras hipótesis afines. 
para describir y ordenar las múltiples formas que la migración internacional contiene, sino también para apoyar el proceso de creación de políticas públicas, de cara a la gestión y administración de un fenómeno que parece tomar cada vez más relevancia en medio del proceso de globalización contemporáneo.

Así, este artículo busca partir del marco metodológico de los sistemas de migración para analizar la forma, el alcance y las limitaciones del desplazamiento humano desde, a través de y hacia Colombia en la actualidad. El argumento es que el fenómeno migratorio, multidireccional y poliforme en el caso colombiano, más allá de responder a fenómenos económicos o políticos, es también ampliamente permeado por líneas de penetración cultural. Lo anterior implica la necesidad de acercarse a un modelo teórico que complemente las explicaciones existentes hasta el momento.

Así, este texto aborda en la primera parte el concepto de sistema de migración según Bakewell. En la segunda parte, este será adaptado al caso de estudio con el propósito de caracterizar la formación de un sistema de migración colombiano en la historia reciente. Posteriormente, se abordarán las conclusiones que señalan cinco temas puntuales surgidos del análisis, dirigidos a la definición de políticas públicas al respecto.

\section{EL CONCEPTO DE SISTEMA DE MIGRACIÓN}

Bakewell (2013), en su artículo "Relaunching Migration Systems", propone un marco de estudio para los procesos migratorios internacionales, partiendo de una revisión de la literatura existente sobre sistemas sociales. Un sistema es entendido como un conjunto de elementos que interactúan entre sí de una forma más o menos armónica, generando resultados -outputs- concretos (Bakewell, 2010). En el estudio de la migración, esta sería una forma de organizar, conceptualizar y entender el fenómeno desde una óptica poliforme y multidisciplinar. Así define sistema de migración con base en dos componentes:

Un conjunto de elementos que interactúan entre sí, incluyendo flujos de personas, ideas y bienes; instituciones en el sentido de discursos y prácticas asociadas (como la “cultura de migración”, el tráfico [de personas] y la desigualdad); y estrategias, como planes de acción de actores particulares (por ejemplo estrategias individuales o de los hogares, políticas de los gobiernos, negocios privados, y organizaciones de la sociedad civil) relativas a la migración entre localidades y

Las dinámicas que gobiernan [o articulan] la forma en que estos elementos cambian en relación con los cambios que se presentan tanto en los elementos parte de estos sistemas (mecanismos de retroalimentación) como en el entorno (Bakewell, 2013, p. 11, traducción del autor).

Si se explora esta propuesta de definición desde las relaciones internacionales, esta parece tener sentido de cara a los elementos que hacen hoy que el fenómeno migratorio quepa dentro de su espectro de estudio. Wihtol de Wenden (2013), haciendo alusión a la transición en dicha disciplina, lo expone de la siguiente forma: 
...los estudios que se hacían hacia arriba, hacia la "gran política", fueron sustituidos por los estudios hacia abajo, hacia el análisis de las sociedades comparadas, y hacia nuevos objetos económicos, sociales y culturales consolidando [a la migración internacional] como un tema de estudio legítimo dentro de las relaciones internacionales (p. 67).

En esta lógica de "arriba hacia abajo", el enfoque sistémico de Bakewell traería consigo ciertas ventajas. La primera tiene que ver con la exploración del fenómeno migratorio por fuera del nacionalismo metodológico ${ }^{2}$ (Vertovec, 1999; Wimmer y Glick-Schiller, 2003) fuente de limitaciones explicativas frente a lógicas internacionales contemporáneas. La segunda es una ventaja de orden práctico. El modelo sistémico se presta para organizar y referenciar procesos culturales que hacen parte del proceso migratorio, tan importantes como las esferas de análisis tradicionales, tanto económicas como políticas.

Dicho esto, se comienza a indagar por la formación del sistema de migración colombiano. Se tienen en cuenta así flujos, instituciones y estrategias que surgen en el marco de la migración desde, a través de y hacia Colombia. Esta asociación se construye más a partir de la observación de las interacciones sociales de los migrantes con su origen y destino que a partir de las estadísticas.

También se consideran los determinantes de la migración y los principios que delimitan esta decisión (Massey et al., 1993). En esta categoría caben tanto el contexto económico como el político, pero también procesos asociados a la reproducción del conjunto social, la tradición del proceso migratorio en una comunidad determinada y su importancia como experiencia personal o grupal, entre otros factores. Un ejemplo de ello es el entendimiento de la migración como rito de paso en un grupo social determinado.

Además, hay que tener en cuenta el carácter evolutivo de los sistemas. Los flujos, las instituciones y las estrategias, articulados a través de las dinámicas que los conforman, no son entidades estáticas en el tiempo; su forma puede ir mutando dependiendo de los hechos que tengan lugar. La renegociación de los términos de la migración, el surgimiento de diferentes formas de acción y conducta dependientes del cambio cultural, las mutaciones en la dirección y el ejercicio del poder, entre otros, así lo determinan (Tomei, 2011). Esto le abre paso a los mecanismos de retroalimentación-o perpetuación-que Bakewell sugiere.

El sistema se alimenta principalmente de flujos de personas desde, a través de y hacia el territorio colombiano, así como de las ideas y los bienes asociados a estos. La dirección del flujo cambia dependiendo de su objetivo. En el caso de la emigración y la inmigración -lo cual incluye el retorno-, el fin último del migrante es en principio asentarse en el lugar de

2 Este concepto crítica las metodologías de las ciencias sociales que ubican al Estado-nación como contenedor automático de datos, voluntades y procesos, que limita el espectro de análisis de la migración transnacional. Ver GlickSchiller, Basch y Szanton Blanc (1995). 
destino. En el caso del tránsito, el espacio donde este proceso tiene lugar o bien sirve como un trampolín necesario para aminorar costos de transacción o bien es el resultado de un proceso que no puede ser llevado a cabo de otra forma. Aquellos flujos que constituyen la movilidad humana contemporánea, que se refieren al turismo o a viajes de negocios (Koser, 2010), hacen parte del sistema por asociación.

\section{LA FORMACIÓN DEL SISTEMA DE MIGRACIÓN COLOMBIANO}

\section{Flujos, instituciones y estrategias en el caso colombiano}

Para explorar la formación del sistema de migración colombiano hay que partir de la premisa más o menos compartida por la literatura, de que durante los últimos cincuenta años Colombia se ha perfilado como un país de emigración ${ }^{3}$. Por una parte, dicha asociación es el resultado de la expulsión constante de personas, derivada de una multiplicidad de causas que, en cualquier caso, van más allá de factores económicos o políticos, y que se podrían catalogar según Bakewell como discursos y prácticas, es decir, instituciones. Esto depende, por ejemplo, de la desigualdad inherente a la estructura socioeconómica del país que impacta el mercado laboral y del momento del ciclo económico.

Esto se suma a la percepción de inseguridad producida por el conflicto armado y sus elementos conexos, que se han convertido en principios que constriñen las actividades y expectativas de migrantes prospectivos. Lo anterior ha producido que a través del tiempo se generen tradiciones de emigración, calificables también como instituciones según el modelo. Estas son más marcadas en algunas zonas del país que en otras, no solo en el rango de las fronteras venezolana y ecuatoriana -muy dinámicas en términos migratorios-, sino también en áreas que han sido históricamente proclives a la emigración ${ }^{4}$.

Si se tiene en cuenta la periodización que hace la literatura sobre las oleadas de emigración colombiana, se pueden identificar tanto instituciones como estrategias base de este sistema de migración. En la base del análisis está la primera ola de emigrantes colombianos, que entre los años sesenta y principios de los setenta tuvo como destino principal Estados Unidos y que se convirtió en el mito fundacional de la

3 Antes de la Segunda Guerra Mundial, el país recibió a varias “comunidades históricas” de inmigrantes. Entre estas se destaca el flujo de sirio-libaneses que se asentó en el país entre el último cuarto del siglo XIX y 1914, algunas comunidades de europeos en el mismo periodo (alemanes en Santander, judíos de diferente proveniencia en el Viejo Caldas y Antioquia) y otros flujos menos significativos en el periodo 1930-1945. Sin embargo, estos flujos no se comparan de ninguna forma con aquellos recibidos por los países del Cono Sur, Brasil, Venezuela o Perú, en donde la concentración histórica de inmigrantes ha sido mucho mayor que en Colombia.

4 Algunas comunidades emigrantes del Eje Cafetero y Antioquia, por ejemplo, son estudiadas por Cruz et al. (2008). 
diáspora colombiana contemporánea ${ }^{5}$ (Cárdenas y Mejía, 2006; Aya et al., 2010).

En la configuración temprana de dicha diáspora influyó la búsqueda del sueño americano, una institución del sistema si se tiene en cuenta su valor discursivo y su poder de atracción para migrantes y no migrantes. Este acercó a trabajadores de alta y no tan alta calificación a la idea de acceder a más y mejores oportunidades laborales en destino (Arango, 2000). También tuvo que ver la orientación de una política de reclutamiento dirigido en destino -derivada del cambio en el sistema de cuotas de inmigración en Estados Unidos(Portes y Rumbaut, 2006), que actuó como una estrategia sistémica temprana. Al tiempo, se tejieron redes sobre las cuales se comenzó a construir el imaginario contemporáneo del inmigrante colombiano ${ }^{6}$-otra institución-y con estas surgieron incentivos para utilizar canales irregulares, una vez saturadas las opciones regulares, como estrategias de emigración alternativas.

El imaginario del migrante que afronta y sortea la adversidad ante las muchas circunstancias desfavorables que el destino le depara, sigue vigente hasta hoy pese a estar ya presente en el espíritu de la segunda ola de emigración. Esta se constituyó durante los ańos setenta y ochenta, con decenas de miles de colombianos que, además de seguir los pasos de los pioneros en Estados Unidos, llegaron también en masa a la pujante Venezuela en plena expansión petrolera (Álvarez de Flórez, 2004). Se establecieron canales de flujos de ideas y bienes a través de la frontera, originados en la dinámica migratoria que se mantiene hasta la fecha.

Luego, ante las difíciles condiciones de seguridad, acompañadas de la profunda crisis política y económica de la década de los noventa, se formó una tercera ola de emigrantes -la más robusta cuantitativamente hablando- (MejíaOchoa, 2012). Sus miembros retomaron muchas veces nexos con familiares, amigos y conocidos de vieja data radicados en el exterior. La emigración se fortaleció como institución que se activa en momentos de crisis y el uso de redes se convirtió en estrategia de movilidad.

Esta tercera ola diversificó sus destinos, lo que trajo como consecuencia la extensión de la diáspora hacia Europa, especialmente a Espańa, así como a lugares no tradicionales en América Latina (Echeverri, 2005; Muñoz de Bustillo y Antón, 2013). En esto tuvieron que ver las estrategias utilizadas en el momento de migrar, lo que llevó a muchos a valerse de acuerdos multilaterales de movilidad en Suramérica o a la búsqueda de una residencia permanente en España luego de dos años de permanencia legal ${ }^{7}$.

\footnotetext{
5 Siguiendo a Fleischer (2012, p. 73), basado en McKeown, una diáspora es "una comunidad de migrantes disímiles" que se teje a través de "las conexiones, redes, actividades y conciencias globales que relacionan puntos de referencia locales" y territoriales.

6 La teoría de redes en el estudio de la migración transnacional asume que los nodos y las conexiones creados por los migrantes y no migrantes en diferentes locaciones terminan perpetuando el fenómeno, más allá de determinantes Push and Pull tradicionales en el estudio de la migración. Ver Solimano (2010).
} 
Los diferentes flujos, instituciones y estrategias moldearon así, tanto numérica como conceptualmente, la tradición diaspórica colombiana en el extranjero. Esta se articula hoy con el lugar de origen a través de redes transnacionales conformadas por migrantes e instituciones de migración, que facilitan la difusión de información para los migrantes prospectivos, de cara al mercado laboral, las características de la sociedad receptora o los pormenores del alojamiento y estadía (De Haas, 2008; King, 2013).También, se convierte en causa del origen y de la perpetuación del fenómeno en muchos casos. Hoy, aunque los incentivos tradicionales -económicos y por factores de seguridad-que estuvieron en la base de dichas olas emigratorias persisten, estos también se han diversificado ${ }^{8}$.

La idea de que Colombia es un país de emigración se ha alimentado de las acciones de la diáspora, activa a través del envío de remesas y cierta presencia política en Norteamérica, el vecindario latinoamericano y en menor medida en Europa occidental ${ }^{9}$. Pero en esto son también importantes las diferentes imágenes, hechos e incluso mitos de diferente naturaleza, que constituyen prácticas discursivas muy similares a lo que Bakewell llama instituciones. Existen imaginarios como el del migrante $p a i s a^{10}$ que se piensa se encuentra en virtualmente cualquier lugar del mundo. También habría que pensar en procesos económicos, como la creación de líneas de negocio en Colombia y el exterior dirigidas a la diáspora, visibles, por ejemplo, a través de canales de televisión por cable en los que pautan constructores de villas a las afueras de ciudades del país pagables en dólares o euros, pensadas para el buen retiro del trabajador inmigrante ${ }^{11}$.

Existen también nichos comerciales étnicos en los que la diáspora tiene gran participación. Esto va desde la compra y venta de bienes de consumo masivo producidos en Colombia ${ }^{12}$, hasta la especialización en negocios propios, legales e ilegales, típicos de "ciudades globales" (Sassen, 2005), como los rickshaws londinenses ${ }^{13}$ o las tristemente célebres cadenas de proxenetismo en algunas zonas de Europa y Asia. También se incluyen iniciativas públicas y privadas como "Colombia nos une"14, "Es tiempo de volver"15

El dinamismo de este acuerdo se ha visto afectado desde la crisis económica de 2009.

8 Hoy, entre 3'300.000 y 5'500.000 colombianos - dependiendo de la fuente- viven fuera del país, lo que constituye entre el 8 y el 13\% del total de la población colombiana. Cfr. Ramírez y Mendoza (2013); dane (2006).

9 Keck y Sikkink (1999), explicarían lo anterior a través del concepto de Transnational Advocacy Network,

10 Persona originaria de las regiones de Antioquia y el Eje Cafetero colombiano.

11 Los ejemplos se encuentran en el espacio publicitario de canales como Telecolombia y Caracol Internacional.

12 Algunos ejemplos anecdóticos son la venta de pantalones "levantacola”, talco para pies y guascas para ajiaco, entre otros.

13 En esta ciudad se han identificado al menos dos empresas de rickshaws (bicitaxis) de propiedad colombiana, que contratan latinoamericanos masivamente, compitiendo en un negocio históricamente perteneciente a migrantes del sudeste asiático.

14 Programa avalado por la Cancillería colombiana. Disponible en: http://bit.ly/1F7eXUO 
o la misma "Red Caldas"16 que están dirigidas en algunos casos a mejorar las condiciones de vida de los emigrantes, pero también a cooptar el capital humano de la diáspora.

El sistema de migración surgió, entonces, en medio de la concepción de que Colombia, Estado y nación, económica y socialmente, ha sido un país de emigración durante el último medio siglo. Sin embargo, desde hace poco más de una década, esto parece estar cambiando. Los números han comenzado a revertir la tendencia emigratoria. Las tasas netas de migración de 2001 a hoy -con la excepción de 2009- han mostrado cómo la brecha entre las personas que entran y salen ha comenzado a reducirse, al tiempo que el stock de extranjeros comienza lentamente a ensancharse (Ramírez y Mendoza, 2013). Esto ha forjado nuevos flujos, instituciones y estrategias que perfilan a Colombia como un lugar de tránsito, retorno e inmigración (figura 1).

FIGURA 1

EVOLUCIÓN ESTIMADA DEL PATRÓN DE EMIGRACIÓN E INMIGRACIÓN EN COLOMBIA

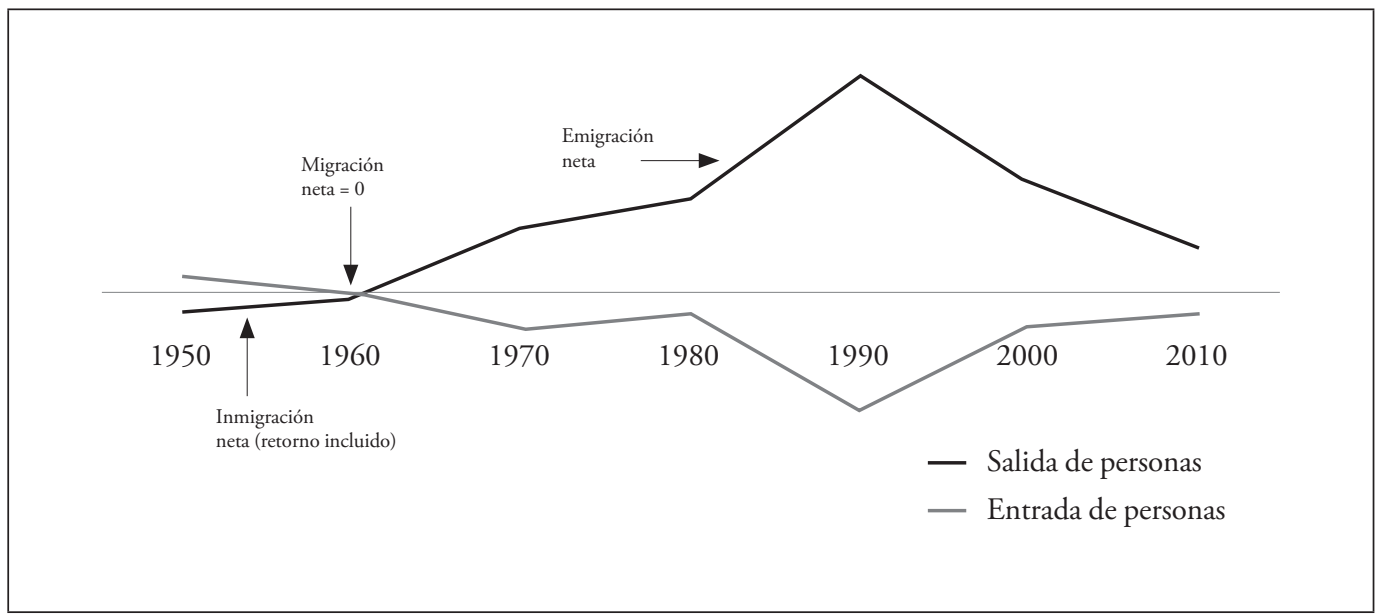

Fuente: proyección del autor basado en DANE (2006, 2014); Cárdenas y Mejía (2006); Mejía-Ochoa (2012).

15 Programa avalado por el Departamento Administrativo de Ciencia, Tecnología e Innovación (Colciencias) del Gobierno colombiano.

16 Red de investigadores colombianos en el exterior, activa desde la década de 1990 bajo el auspicio del Gobierno colombiano y el sector privado. Disponible en: http://bit.ly/19x82Jb 
Lo anterior se basa, en parte, en la observación estadística. En el perfil migratorio del país elaborado por Ramírez et al. para la Organización Internacional para las Migraciones (OIM) (2014), se menciona que según el Banco Mundial, cerca de 110.000 extranjeros residirían en Colombia (p. 65) -casi $200 \%$ más que en 1999- mientras que se han concedido más de 40.000 permisos de residencia para no nacionales que buscan hacer negocios en el país (p. 68) ${ }^{17}$. Esto, sin debatir el problema derivado de la imposibilidad de contabilizar los colombianos que han adquirido su nacionalidad por adopción, nacidos en el extranjero. No obstante, en estos números se incluyen comunidades "históricas" de inmigrantes, provenientes de países vecinos, algunos países de Europa y un par del Medio Oriente. Pero los indicadores se construyen también a partir de flujos conformados por una nueva ola de inmigrantes, que ha comenzado a llegar a Colombia a partir de la segunda mitad de la década de 2000 (Migración Colombia, 2014) ${ }^{18}$.

Sobre los determinantes del arribo de inmigrantes -en tránsito y con el ánimo de residir-hay que tener en cuenta una amplia gama de elementos que pueden ser vistos como parte del enfoque sistémico. Varios han llegado por trabajo, unos más por estudio, algunos otros por azar, otros haciendo parte o siendo víctimas de redes de tráfico ilegales y muchos en tránsito hacia otros destinos, que buscan llegar a Panamá utilizando la ruta colombiana como estrategia -donde la política de refugio y asilo es más flexible que en Colombia- o Estados Unidos-que sigue siendo un polo de atracción laboral indiscutible- (Stanton, 2014) ${ }^{19}$.

Hay instituciones en plena formación, asociadas a la idea de que Colombia atraviesa por un momento de expansión económica, en lo que ha influido en mayor o menor forma el proceso de lavado de la imagen del país en el contexto internacional. Pero también influye el flujo de información entre nodos de inmigrantes. En esta se incluyen las condiciones del mercado laboral para personas mediana y altamente calificadas o algunos datos sobre el trato diferenciado a los extranjeros en el país -sobre todo si son de origen europeo y norteamericano-- Se informa, además, la existencia de una serie de bienes y servicios tangibles e intangibles a los que se tiene acceso restringido en origen, que van desde el precio del servicio doméstico, la temperatura constante durante todo el ańo o el acceso a salud privada de alto nivel a precios cómodos.

En la lógica del tránsito se gestan flujos, instituciones y estrategias particulares de esta

17 El diario El Tiempo publicó en su primera página del 22 de septiembre 2014 que "Cada día, 116 extranjeros obtienen visa de trabajo en Colombia” (p. 1).

18 Evidencia parte de un avance académico por publicar, el cual argumenta que hoy se pueden encontrar en ciudades del país, incluso intermedias, familias iraníes o un grupo de adolescentes turcos en el supermercado, una empleada argentina trabajando en el restaurante propiedad de un mexicano, un grupo de veinteańeros cubanos buscando asilo, una colonia de comerciantes chinos en los Llanos Orientales y un par de almacenes de artículos "persas" de propiedad bangladesí.

19 Las FARC y otros grupos al margen de la ley estarían sacando réditos de este flujo (Blu Radio, 2015). 
condición estacionaria. En ella hay una interacción con la sociedad de destino de diferente naturaleza, en un espacio donde migrantes y no migrantes interactúan en pro de un fin propio (Masferrer, 2012). Se han identificado, por ejemplo, rutas preferidas por asiáticos y cubanos en las que los intermediarios locales se han encargado de proveer servicios como transporte y alojamiento, específicos según grupos nacionales. Otro elemento asociado es el trámite de extensiones de estadía en las oficinas de Migración Colombia, en las que al parecer se han formado nichos de negocio ilegal, entre otros (González, 2014).

La temporalidad de la estadía afecta también este proceso. Algunos inmigrantes se quedan solo por meses, semanas o días, pero logran configurar experiencias migratorias completas, en tanto existe una articulación entre flujos, instituciones y estrategias. Esto es experimentado también por aquellos que se quedan por periodos más amplios de tiempo e incluso por los que terminan quedándose irregularmente de manera permanente ${ }^{20}$. Además, la facilitación del transporte internacional, que incluye la reducción en los costos y la diversificación de los medios y las rutas son factores que han conducido a que los flujos internacionales de migrantes tiendan a amplificarse y a que sean mucho más dinámicos que en el pasado. De esta manera, se han formado flujos circulares $^{21}$, de los que también participan colombianos, como parte del sistema.

Por último, este proceso se ha construido en un contexto en el que la crisis financiera internacional iniciada en 2008 -y en algo el crecimiento económico del país- ha permitido que surjan condiciones para el retorno de muchos nacionales que se habían asentado en el extranjero. Lo anterior habría estimulado la creación de programas destinados para tal fin por parte de los gobiernos en origen y destino, que tienen un efecto en la percepción del migrante sobre sus propias condiciones.

En esto influye la situación del mercado laboral en las sociedades de destino, así como la apertura de posibilidades de inversión, negocio y trabajo en Colombia. A su vez, hay que tener en cuenta que la mayoría de estos retornados serían parte de la denominada tercera ola migratoria, lo que los hace contar con nexos y relaciones mucho más profundas y frescas en origen si se compara con emigrantes de las demás oleadas. Esto, en últimas, termina por aminorar los costos tangibles e intangibles en los que se incurre durante el retorno, activando instituciones y estrategias presentes en el modelo. La combinación de retornados y nuevos inmigrantes explica la contracción de la brecha entre entradas y salidas netas del país que conforman la tasa de migración neta de la cual se hablón 22 .

20 Pese a la dificultad para llegar a cifras fiables, al respecto hay indicios de este fenómeno. Para 2007, algo más de 2500 ciudadanos chinos habrían permanecido en el país de esta forma (UNODC, 2014), mientras que existen testimonios de migrantes irregulares que hablan de su permanencia en Colombia a través de este mecanismo (Serna, 2015; HsB Noticias, 2015).

21 Flujos de ida y vuelta -e intermedios- emprendidos por un migrante o grupo de migrantes (Vertovec, 2007). 


\section{Las dinámicas normativas del sistema}

A partir del enfoque de Bakewell, las dinámicas de un sistema migratorio se pueden dividir en normativas y en aquellas delimitadas por el entorno. Las primeras, se asocian con elementos que desde las esferas normativas formales e informales articulan flujos, instituciones y estrategias del proceso. Las segundas, hacen referencia al contexto en el que aparece el sistema, que dependen de las características particulares de los procesos migratorios así como de las externalidades que lo afectan y que terminan retroalimentando -o perpetuandosu existencia.

Entre las dinámicas normativas formales del sistema de migración colombiano se encuentran leyes relativas a la migración, la nacionalidad y extranjería, y la pertenencia y debida obligación del Estado con organizaciones internacionales, convenciones, acuerdos y tratados internacionales. Pero también, es necesario ver los procedimientos informales que hacen parte del sistema, como la tradición, la costumbre, la coyuntura económica y políti$\mathrm{ca}$, la lógica de mercado y la cultura del orden social dominante.

Estos mecanismos no se limitan a un único elemento dentro del modelo. Muchas veces, una normativa formal hace parte de las instituciones pero también de las externalidades que limitan o amplifican una estrategia en el sistema. En otros casos, los mismos flujos, como la información, sirven como canales sobre los que se extiende una institución de migración.

Pensando en los compromisos adquiridos en el nivel internacional, Colombia hace parte de la Organización Internacional para las Migraciones desde 1956 -antes Comité Intergubernamental para las Migraciones Europeas-(OIM, 2014) y cuenta con varios acuerdos relativos a la libre movilidad de ciudadanos con miembros de estructuras como la CAN y otros acuerdos bilaterales con países de la región ${ }^{23}$. A su vez, el Gobierno colombiano ratificó el Convenio de Ginebra sobre Refugio y Asilo en 1961 y se adhirió a su protocolo adicional en 1980 (Acnur, 2011).

Además, derivado del ingreso del narcotráfico en la agenda de seguridad internacional durante los ańos ochenta -en gran medida- y de la percepción sobre Colombia como exportador neto de migrantes - en menor medida-, sus ciudadanos necesitan un visado para cruzar fronteras en buena parte del mundo ${ }^{24}$. Estos requisitos generan la necesidad de canales para emigrar por fuera de los marcos formales establecidos, lo cual hace que la migración

22 Para ilustrar esto se pueden procesar datos de Migración Colombia (2012, 2013, 2014). La evolución de la tasa de migración neta habría caído de un 14, 58 \% en marzo de 2012 a un 11,87\% en marzo de 2013 y a un 10,22\% en marzo de 2014.

23 Hoy, la discusión se centra en la negociación de un área de libre movilidad dentro del espectro geográfico de los países miembros de la Alianza del Pacífico: Chile, Colombia, México, Perú y, eventualmente, Costa Rica.

24 Desde 2012, se han logrado acuerdos con gobiernos como el turco y el ruso para el levantamiento de este requisito. Además, se espera la liberalización de la entrada de colombianos al espacio Schengen a partir de algún momento de 2015. 
irregular se convierta a la vez en institución y estrategia constante del sistema de migración colombiano.

En el contexto interno, los últimos años se han caracterizado por la suscripción de varios acuerdos y normativas que buscan actualizar la administración del asunto migratorio. Lo anterior ha tenido efectos sobre el fomento de estrategias de emigrantes e inmigrantes, pero también sobre la formalización de instituciones sociales de migración.

Este ordenamiento sistémico atraviesa por tres ejes. El primero tiene que ver con el control de la salida y entrada de personas del territorio colombiano - eje transversal, administrado hoy por la agencia Migración Colombia- (Ministerio de Relaciones Exteriores, 2014b). El segundo se relaciona con el Asilo Político-figura híbrida propia del sistema latinoamericano "establecido por no menos de diez tratados sobre asilo y extradición”- (Franco, 2012, p. 12). El tercero se sostiene de la asignación de visas de trabajo temporal, de cónyuge, religiosas -entre otras y que regularizan la situación de los extranjeros en el país-, proceso hoy administrado por el Ministerio de Relaciones Exteriores.

También, conforme el fenómeno migratorio ha adquirido cierta relevancia en la política central desde la década del 2000, se ha generado cierto interés por gestionar formalmente el asunto de la diáspora colombiana. La motivación primordial para hacer esto tendría un doble componente: el interés del Gobierno central por gestionar los ingresos que recibe el país por concepto de remesas y el capital electoral representado en los votos de estas comunidades en el extranjero (Cruz et al., 2008).

El cambio de postura del Gobierno frente a las remesas se potenció a partir de informes del Banco de la República sobre el progresivo incremento de la participación de estas en el PIB (Banco de la República, 2015) ${ }^{25}$. Esto trajo consigo el posicionamiento de la diáspora en la agenda. En 2003, se creó el programa Colombia nos une, con el objetivo de "generar mejores condiciones a los colombianos que residen en el exterior" (onu, 2014). Desde 2011, este programa depende de la Dirección de Asuntos Migratorios del Ministerio de Relaciones Exteriores.

Hoy, convertido en departamento, el programa cuenta con un paquete de apoyo a los miembros de la diáspora-con capacitaciones, asistencia técnica y promoción cultural- que funciona en zonas de alta concentración de colombianos en Estados Unidos, Espańa y el Reino Unido. Este cuenta también con un plan de acompañamiento al retorno que es en buena medida el resultado de presiones generadas por compromisos que el Gobierno ha adquirido en el nivel internacional -con Espańa, por ejemplo-, como condición para apoyar el acuerdo de asociación económica con la UE que incluiría la liberación del visado Schengen para nacionales colombianos. Cuenta también con un plan de inmigración ordenada y regulada orientada al retorno y un plan para colombianos destacados con un propósito similar (Ministerio de Relaciones Exteriores, 2010). 
No hay que olvidar las presiones electorales asociadas a la creación de estas normativas. Terminando el segundo periodo del Gobierno Uribe, surge en 2009 el documento Conpes ${ }^{26}$ 3603 denominado "Política integral migratoria”, el cual orienta hasta hoy la gestión del asunto migratorio (Ministerio de Relaciones Exteriores, 2010). Posteriormente, el Gobierno Santos ha sido el ejecutor de los lineamientos establecidos en este documento. En 2011, se creó por medio del Decreto Presidencial 4062 la Unidad Administrativa Especial Migración Colombia que, como se comentó, es la unidad que administra el ingreso y la salida de personas del territorio colombiano.

El mismo año se sancionó la Ley 1465 , que adoptó el nombre de Sistema Nacional de Migraciones impulsado, entre otros, por el movimiento político MIRA, que cuenta con una notable presencia entre las comunidades colombianas emigradas en Estados Unidos (Congreso Visible, 2014), que reivindica una mayor participación política de la diáspora. Otros elementos de esta normativa incluyen el mandato dado a Colciencias para cooptar a colombianos altamente calificados en el exterior -y que hoy se agrupa bajo el programa "Es tiempo de volver"-. Otras instituciones, como el Instituto Colombiano de Crédito Educativo y Estudios en el Exterior (Icetex) y Colfuturo, hacen también parte de este universo ${ }^{27}$.

\section{Las dinámicas derivadas del entorno}

Para delimitar el entorno del sistema se sugieren cinco dinámicas puntuales. La primera, tiene que ver con la existencia de una tradición de migración en varias zonas del país. Según cifras del censo general de 2005, las zonas que más emigrantes exportan son Antioquia, Valle del Cauca y el Viejo Caldas - pese a no ser fronterizas-, en donde el retorno impactaría profundamente. Desde la primera ola de emigración se gestaron redes diaspóricas que han llevado a que el flujo se perpetúe en el tiempo y a que los habitantes de estas regiones sean muy cercanos, tanto cultural como económicamente, al proceso migratorio internacional. Esto influye de manera directa sobre la percepción de bienestar, ingreso y oportunidad que se genera en el imaginario de los migrantes y su entorno social, tanto en origen como en tránsito y destino (Alba, 2013). Lo anterior se ha profundizado gracias a la velocidad de las comunicaciones, la facilidad de las interconexiones y de transporte, y la reducción de costos asociados que además terminan facilitando el retorno.

Segundo, es necesario pensar en los determinantes de la migración. La coyuntura económica y la estabilidad política, elementos convergentes en relación con la generación de las tres olas migratorias antes mencionadas, no son ya los únicos aspectos por analizar como

26 Documento elaborado por el Consejo Nacional de Política Económica y Social (Conpes), en el que se determina la orientación de un plan de inversión pública.

27 Colfuturo (Fundación para el estudio de Colombia), es una fundación sin ánimo de lucro apoyada por el Estado y la empresa privada para la capacitación de colombianos en el exterior. 
causantes de los flujos. Dentro de los factores de empuje (push factors) ${ }^{28}$ tradicionales se destacaron las limitaciones del mercado laboral colombiano y la inestabilidad política. Los factores de arrastre (pull factors) tenían que ver en su mayoría con la concepción de que en el lugar de destino las condiciones del mercado laboral eran mejores así como la estabilidad del régimen político en la sociedad receptora.

Hoy, la coyuntura económica internacional ha venido cerrando las expectativas de acceso a los mercados laborales en los destinos tradicionales. Esto ha traído como consecuencia la diversificación de los destinos de migración así como el retorno de varios grupos de migrantes. Sin embargo, si bien el país pareciera contar a 2015 con una capacidad de absorción laboral más amplia que en tiempos pasados, aún dista mucho de proporcionar algunos de los beneficios tangibles e intangibles que se les han ofrecido a los antiguos migrantes en su origen o antiguo destino, pese a ofrecer su propia gama de intangibles a aquellos que llegan o vuelven. Por otro lado, derivado en parte del proceso de "lavado" de imagen internacional basado en los resultados macroeconómicos y en seguridad defendidos por su Gobierno, Colombia se ha convertido para ciertos grupos de extranjeros en una posibilidad de destino -aunque no siempre la primera- (Brodzinski, 2014; Serna, 2015).

Este asunto se enmarca también en el cambio de las aspiraciones y expectativas de quienes migran. Desde la época de Ravenstein $^{29}$, y hasta hoy (Castles et al., 2013; Massey et al., 1999; Hatton y Williamson, 1998), la teoría de migración ha prescrito que quienes migran de manera no forzada, no son los individuos más pobres de una sociedad, sino aquellos que tienen la capacidad de acumular el capital necesario para cubrir los costos mínimos del desplazamiento inicial. En el caso colombiano esto es cierto, sobre todo para aquellos pioneros de las primeras dos olas de emigración, que habrían sido motivadas mayoritariamente por condiciones económicas.

Si esta característica se pone en el contexto del crecimiento económico que ha experimentado el país durante la última década, y el aparente fortalecimiento y expansión de una clase social con capacidad de consumo y ahorro, hay que pensar en el cambio de las expectativas de estos individuos. Ya el razonamiento básico deja de estar únicamente enfocado en la necesidad de supervivencia, y se abre el espacio para que la motivación de la migración vaya más allá de las razones tradicionales.

La diversificación de la emigración por educación, por nexos afectivos o políticos, por la afinidad con el lugar de destino, la emigración por el descontento con el orden cultural en origen, por la expectativa de adquirir una experiencia migratoria, entre muchos otros factores, adquiere entonces relevancia en este sistema. Esto afecta el nivel del entendimiento cultural, que a su vez influye en el diseño de

28 Los factores de empuje ( $p u s h)$ y arrastre ( $p u l l)$ hacen parte de la teoría clásica de la migración humana impulsada por Lee (1966).

29 Primer teórico moderno de la migración humana, quien hizo sus observaciones en la Inglaterra del siglo XIX. 
las instituciones y estrategias de las que habla Bakewell, pero sobre todo en las dinámicas que gobiernan la migración.

Tercero, y muy arraigado a la marcada concepción cultural de unidad familiar, hay que tener en cuenta la forma que toman las remesas en Colombia, y cómo esta institución es más que un flujo de capital. Para muchos emigrantes, la decisión de migrar hace parte de una estrategia de supervivencia familiar-al estilo de la explicación proporcionada por la teoría de la nueva economía de las migraciones- (Arango, 2000). Aquellos más capaces dentro de la célula social terminarían por migrar como estrategia de supervivencia para su núcleo familiar, haciendo de las remesas un elemento central de la experiencia migratoria. Visto desde un punto de vista puramente sociológico, la proporción del ingreso del migrante destinada a las remesas en el caso colombiano, superior al promedio mundial (IDB, 2013), puede tener varias lecturas ${ }^{30}$.

Por una parte, tendría una relación directa con el alto valor social que tiene la acción de enviar dinero a casa, lo que a su vez concuerda con la alta participación de las remesas en el PIB colombiano. Los vínculos afectivos hacen que la remesa sea un mecanismo que muchas veces define los términos de las relaciones familiares, tanto por una cuestión de deber como de prestigio (Collier, 2013). Este es un elemento que ha permanecido más o menos constante hasta hoy, pero que se ha visto afectado por la crisis financiera internacional. Por otra parte, una porción de las remesas -resultado parcial de la percepción cultural sobre el dinero- se va al consumo del núcleo familiar (Leal-Calderón, 2008; Roa, 2010). Esto tiene que ver también con las dinámicas de prestigio en la región de recepción de la remesa.

La naturaleza de las remesas ha venido cambiando conforme la dirección de los flujos se ha transformado. Si bien es cierto que los flujos hacia Colombia siguen abarcando la gran mayoría de las remesas privadas, también hay que pensar en los flujos de salida. Puede que los individuos que envían y reciben dinero hacia y en el exterior sean menos que los que envían y reciben dinero hacia y en Colombia. No obstante, las cantidades alcanzan a ser bastante significativas, más si se tiene en cuenta su evolución en años recien$\operatorname{tes}^{31}$. En esto habría que tener en cuenta tanto a extranjeros que envían dinero a sus lugares de origen como a aquellos colombianos que envían remesas a miembros de su entorno familiar (hijos y otros dependientes). Muchos de ellos harían parte de capas económicas que tienen mayor capacidad de ahorro e inversión, lo que los pone en capacidad de enviar montos significativos, en respuesta a una necesidad económica que cuenta también con un significado social en términos de deber y prestigio.

\footnotetext{
30 Se ha registrado que luego de garantizar los ingresos para su supervivencia básica, el migrante colombiano comienza a acumular el monto necesario para completar su remesa, antes que destinar este ingreso para consumo suntuario (IDB, 2013; Montoya et al., 2010).

31 Según datos del Banco Mundial (2011), entre 2006 y 2009 desde Colombia se enviaron al exterior más de 250 millones de dólares. Para el periodo comprendido entre 2011 y 2014, esta cifra habría aumentado a unos 300 millones de dólares (Banco de la República, 2015).
} 
Como cuarto punto hay que tener en cuenta el momento por el que atraviesa el asunto de la relación migratoria entre Colombia y Venezuela. En el pasado reciente, esta relación se inscribía bajo la lógica unidireccional del origen de emigración-Colombia- hacia el destino de inmigración -Venezuela-. Hoy, derivada de diversos factores políticos, económicos y sociales esta es una relación migratoria de doble vía. Si bien la tendencia no se ha revertido completamente en términos nominales, ambos son al tiempo países de recepción y emisión de migrantes. De hecho, muchos colombianos de la zona de frontera y de la costa atlántica ven como un pull factor los subsidios que el Gobierno venezolano está en disposición de proveer para bienes de consumo o vivienda -entre otros- a trabajadores menos calificados ${ }^{32}$. Al tiempo, muchos venezolanos de clase media y media-alta, con niveles importantes de capital humano, encuentran en Colombia una opción de residencia, por razones relacionadas con la seguridad institucional, la percepción de la política en su país y la saturación de venezolanos en otros destinos -como Estados Unidos o Panamá-.

Este es un punto de quiebre en la consolidación del sistema migratorio colombiano contemporáneo que comienza a influir en cómo se articulan los flujos, las instituciones y estrategias asociadas ${ }^{33}$. Esta dinámica es la manifestación de un proceso migratorio que abarca una multiplicidad de determinantes, que genera relaciones sociales profundas a través de la extensión de las redes y que, en últimas, termina teniendo efectos políticos decisivos en la agenda bilateral. Más allá del debate ideológico por el que atraviesa el tema desde el Gobierno venezolano, la densidad del proceso hace que el tema migratorio en ambos países deba pasar por la evaluación del efecto de los flujos hacia y provenientes de su respectivo vecino.

La última dinámica que gobierna el sistema en cuestión se asienta sobre la idea compartida por parte de la diáspora colombiana de volver al origen en el momento del retiro. Este "anhelo de retiro" ha hecho parte del sistema de migración colombiano desde su configuración en la segunda mitad del siglo xx. Sin embargo, en el último tiempo, la ocurrencia del retorno se ha venido incrementando en tanto las condiciones económicas, tecnológicas y de transporte lo han hecho posible (Dustmann y Weiss, 2007). Hoy en día, se ha incrementado la probabilidad de que el migrante colombiano se retire en su país de origen. Así, hay un contraflujo de migrantes retornados que llegan -en parte- con cierto poder de consumo e inversión proporcionado por las pensiones que reciben y el ahorro generado en sus antiguos países de residencia.

Ahora bien, el impacto en la sociedad de retorno debe ser analizado más allá de los puros elementos económicos. La capacidad de adap-

32 Sobre esto existe un intenso debate que imposibilita llegar a cifras medianamente objetivas (Wallace, 2013; Pardo, 2014).

33 De hecho, Venezuela se había concebido a sí mismo como país de inmigración, lo que ha venido cambiando - de manera relativa- luego de la llegada de Chávez al poder (Dávila, 2000; Pardo, 2014). 
tación y cohesión de un retornado surge de una serie de factores económicos, pero también de otros incuantificables, derivados del impacto cultural (Cheong et al., 2007). En principio, los retornados que duraron menos tiempo en el exterior tendrían una mayor capacidad de readaptación, en tanto han tenido menos tiempo para adoptar el modelo de vida de la sociedad que anteriormente los acogió. También habrían tenido menos espacio para perder sus nexos de afinidad con el origen.

Además, aquellos retornados solteros tendrían en principio la posibilidad de adaptarse de mejor manera. Se han conocido casos de rupturas familiares de migrantes que se han desplazado a Colombia con cónyuges extran- jeros que no se adecuan al país. También hay casos de hijos de colombianos, antiguos emigrantes, que tienen una capacidad limitada de interacción, al no conocer el idioma ni estar familiarizados con el orden cultural prevalente. En este contexto, hay que comenzar a pensar en el posible nacimiento de una generación de familias transnacionales, enmarcada en el sistema de migración colombiano.

Otro elemento importante asociado, es el asunto del refugio y el asilo. Este tema merece una investigación exclusiva. De hecho, se podría asegurar que el sistema propuesto coexiste con un régimen del refugio y el asilo (Nagle, 2004). De cualquier forma, el actual sistema de migración colombiano podría seguir el siguiente esquema:

FIGURA 1.

EL SISTEMA MIGRATORIO COLOMBIANO CONTEMPORÁNEO

\begin{tabular}{|l|l|l|l|l|}
\hline \multicolumn{1}{|l|}{} & Momento migratorio & Flujos & Instituciones & Estrategias \\
\cline { 2 - 5 } & Origen & $\begin{array}{l}\text { Información sobre el destino } \\
\text { y la posibilidad de migrar }\end{array}$ & $\begin{array}{l}\text { Desigualdad en la distribu- } \\
\text { ción del ingreso } \\
\text { Mercado laboral segmentado } \\
\text { Tradición de migración }\end{array}$ & $\begin{array}{l}\text { Diversificación de los ingre- } \\
\text { sos familiares } \\
\text { Acuerdos laborales }\end{array}$ \\
\cline { 2 - 5 } & Tránsito & $\begin{array}{l}\text { Migrantes } \\
\text { Bienes } \\
\text { Servicios } \\
\text { Información }\end{array}$ & $\begin{array}{l}\text { Redes de migrantes en ori- } \\
\text { gen y destino }\end{array}$ & $\begin{array}{l}\text { Empresarios de la migración } \\
\text { Utilización de acuerdos bina- } \\
\text { cionales }\end{array}$ \\
\cline { 2 - 5 } & Destino & Remesas & $\begin{array}{l}\text { Mercado laboral con oferta } \\
\text { de empleo } \\
\text { Subexplotación de las capa- } \\
\text { cidades del migrante } \\
\text { Añoranza del origen }\end{array}$ & $\begin{array}{l}\text { Políticas migratorias } \\
\text { Canales de migración irre- } \\
\text { gular } \\
\text { Nichos de mercado étnico }\end{array}$ \\
\hline
\end{tabular}




\begin{tabular}{|c|c|c|}
\hline \multirow{2}{*}{ 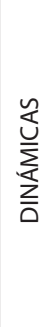 } & Normativas & $\begin{array}{l}\text { Compromisos formales en el espectro internacional } \\
\text { Legislación doméstica } \\
\text { Programas de apoyo a la migración y al retorno } \\
\text { Principios que rigen la dinámica cultural de la migración }\end{array}$ \\
\hline & Derivadas del entorno & $\begin{array}{l}\text { Tradición de migración } \\
\text { Determinantes de la migración } \\
\text { Particularidad de las remesas } \\
\text { Relación migratoria Colombia-Venezuela } \\
\text { Anhelo del retorno }\end{array}$ \\
\hline
\end{tabular}

Fuente: elaboración del autor con base en Bakewell (2010, 2013).

\section{CONCLUSIÓN}

Este texto delineó la extensión, el alcance y los límites del sistema migratorio colombiano. Para esto se argumentó que el fenómeno migratorio en Colombia ha sido permeado por líneas de penetración cultural, más allá de responder a una lógica puramente económica o de seguridad, como lo sostiene la literatura tradicional. En este entendimiento, el concepto de sistemas de migración de Oliver Bakewell fue utilizado como herramienta de organización y abstracción teórica de la información.

Este se establece en medio de la transición de Colombia como país de emigración a uno de inmigración, tránsito y retorno. Se estableció así en una primera parte la definición de sistema de migración para después aplicarla en la segunda parte al caso colombiano. En la estructuración del argumento se tuvieron en cuenta elementos causales, desde la historia reciente, que han terminado por definir los flujos, las instituciones y las estrategias articuladas a través de dinámicas normativas y derivadas del entorno, ejes básicos del análisis sistémico.

La utilidad de este tipo de análisis se refleja en la postulación de ideas al servicio de la generación de política pública en el futuro cercano.
Para esto, sirve pensar en cinco posibles áreas de intervención. La primera, se refiere a la gestión de las remesas con políticas que contemplen la dimensión cultural del asunto. La segunda, se dirige a sacar provecho de las oportunidades que surgen de la pertenencia colombiana a la Alianza del Pacífico. La tercera, se perfila en pro de la reestructuración de los programas dirigidos a la cooptación de colombianos altamente calificados en el exterior. La cuarta, pone de relieve el acercamiento a la diáspora como recurso de poder en el contexto internacional, y la quinta, busca maximizar la gestión de Colombia como país de tránsito. Estos serán los temas de una próxima contribución al respecto (Palma, por publicar).

Al tiempo, quedan muchos otros elementos por incluir dentro de este primer mapeo del sistema de migración colombiano. Algunos de estos tienen que ver con el tratamiento del asunto del refugio y el asilo, la percepción de la migración irregular, desde y hacia Colombia, la trata de personas, la relación entre migración y narcotráfico, el asunto de la migración de estudiantes y otros más. No obstante, este texto impulsa el estudio del fenómeno migratorio en Colombia y la generación de política pública desde un punto de vista pluridisciplinar, basado 
en los estudios internacionales, en constante alusión a la importancia del ordenamiento cultural como mecanismo generador y perpetuador de normas y reglas dentro de una óptica que, tradicionalmente, se estima como puramente económica y política. Este texto sugiere, entonces, la continuación de estudios en esta dirección, que puedan concretar o desestimar las ideas que se han expuesto.

\section{REFERENCIAS}

Acnur (2011). Estados parte de la convención de 1951 sobre el estatuto de los refugiados y el protocolo de 1967. Recuperado de http://bit.ly/1xq9SH1

Alba, F. (2013). Mexico: The new migration narrative. Migration Policy Institute. Recuperado de 2015 de http://bit.ly/1D8dK0i

Álvarez de Flórez, R. (2004). La dinámica migratoria colombo-venezolana: evolución y perspectiva actual. Geoenseñanza, 9 (2), 191-202.

Arango, J. (2000). Explaining Migration: A Critical Review. Oxford: Unesco, Blackwell Publishers.

Aya, M. T., Carvajal, L. y Téllez, G. (2010). Indagación sobre las causas de la escasa inmigración en Colombia: ¿Ausencia de políticas públicas o políticas públicas restrictivas? Opera, 10, 167-183.

Bakewell, O. (2010). Some reflections on structure and agency in migration theory. Journal of Ethnic and Migration Studies, 36 (10).

Bakewell, O. (2013). Relaunching Migration Systems. Migration Studies. doi: 10.1093/migration/ mnt023.

Banco de la República de Colombia (2015). Remesas promedio por país de origen. Recuperado de http://www.banrep.gov.co/es/remesas
Banco Mundial (2011). Datos sobre migraciones y remesas. New York: World Bank Group. Recuperado de http://bit.ly/1HIUrJ4

Banco de la República (2015). Egresos de remesas por país de destino. Recuperado de http://bit. ly/1Fqgt1L

Bermúdez, A. (2011). The "Diaspora Politics" of Colombian migrants in UK and Spain. International Migration, 49 (3),125-143.

Betts, A. (2010). Survival Migration: A New Protection Framework. Global Governance, 16,361-382.

Blu Radio (2015) Tráfico de inmigrantes es el nuevo negocio de las FarC, según la Armada. Blu Radio. Recuperado de http://bit.ly/1DgzcSr

Brodzinski, S. (2014). Bienvenido, Habibi. Foreign Policy. Recuperado de http://atfp.co/1MkbCsp

Cardenas, M. y Mejía, C. (2006). Migraciones Internacionales en Colombia: ¿Qué sabemos? Fedesarrollo. Working Papers Series, 30.

Castañeda, M. (2015). Claramente aún no es tiempo de volver. El Tiempo. Recuperado de 2015 de http:// bit.ly/1DlqKhl

Castles, S., De Haas, H. y Miller, M. (2013). The Age of Migration. London: The Gilford Press.

Cheong, P. et al. (2007). Immigration, Social Cohesion and Social Capital: A Review. Critical Social Policy, 27 (1), 24-49.

Collier, P. (2013). Éxodo: inmigrantes, emigrantes y paises. Madrid: Turner Noema.

Congreso Visible (2014). Por la cual se crea el sistema nacional de migraciones. Recuperado de http:// bit.ly/1CmNkGh

Cruz, P., González, A. y Medina, M. (2008). La diáspora colombiana: derechos humanos y migración forzada Colombia-España 1995-2005. Madrid: Art Cibel Editores.

DANE (2006). Censo General 2005. Recuperado de http://bit.ly/1HVHb8C 
DANE (2014). Estimación de la migración 1973-2005. Recuperado de http://bit.ly/1DgzNUp

Dávila, L. (2000). El impacto social de la migración. En Martínez, J. La migración internacional y el desarrollo de las Américas (pp. 259-275). Santiago: Cepal.

De Haas, H. (2008). Migration and Development. A Theoretical Perspective. University of Oxford International Migration Institute, Working Papers Series, 2008-9.

Dustmann, C. y Weiss, C. (2007). Return Migration: Theory and Empirical Evidence. CReAM Discussion Paper 02/07, University College London.

Echeverri, M. (2005). Fracturas identitarias: migración e integración social de los jóvenes colombianos en España. Migraciones Internacionales, 3 (1), 141-164.

Fleischer, F. (2012). La diáspora china: un acercamiento a la migración china en Colombia. Revista de Estudios Sociales, 42, 71-79.

Fog, L. (2015). Colciencias improvisó con "Es tiempo de volver". El Espectador. Recuperado de http:// bit.ly/1xMqLw3

Franco, L. (2012). El asilo y la protección de los refugiados en América Latina. Buenos Aires: Siglo Veintiuno Editores.

Glick-Schiller, N., Basch, L. y Szanton Blanc, C. (1995). From Immigrant to Transmigrant: Theorizing Transnational Migration. Anthropological Quarterly, 68 (1),48-63.

González, K. (2014). Tres funcionarios de la Registraduría capturados por tráfico de personas. El Heraldo. Recuperado de http://bit.ly/1DgAluz

Gottwald, M. (2004). Protecting Colombian Refugees in the Andean Region: The Fight against Invisibility. International Journal of Refugee Law, 16 (4), 517-546.
Hatton T. y Williamson, J. (1998). The Age of Mass Migration. Causes and Economic Impact. New York: Oxford University Press.

HSBN Noticias (2015). Sueño americano se trunca en Ipiales. Recuperado de http://bit.ly/1dcBXsn

IDB (2013). Remittances in Latin America in 2013: Still below Pre-Crisis Levels. Recuperado de http:// bit.ly/1NrGPVR

Keck, M. y Sikkink, K. (1999). Activist beyond Borders: Advocacy Networks in International Politics. Ithaca: Cornell University Press.

King, R. (2013). Theories and Typologies of Migration: An Overview and Primer. Malmö University, Willy Brandt Series of Working Papers in International Migration and Ethnic Relations, 3/12.

Koser, K. (2010). International Migration and Global Governance. Global Governance, 16 (3), 301-315.

Leal-Calderón, Z. (2008). Las remesas y su relación con el crecimiento económico, el consumo y la inversión: el caso de Colombia. Programa de Economía de la Universidad de Puerto Rico. Recuperado de http://bit.ly/1G5FfZd

Lee, E. (1966). A Theory of Migration. Demography, 3 (1), 47-57.

Masferrer, C. (2012). Going Back Home? Changing Demography and Geography of Mexican Return Migration. Population Research Policy Review, 31 (4), 465-496.

Massey, D. et al. (1993). Theories of International Migration: A Review and Appraisal. Population and Development Review, 19 (3), 431-466.

Massey, D. et al. (1999). Worlds in motion: Understanding International Migration at the End of the Millennium. Oxford: Oxford University Press.

Medina, M. (2014). Hay déficit de 15000 ingenieros para industria. El Espectador. Recuperado de http:// bit.ly/1FZLuiF 
Mejía-Ochoa, W. (2012). Colombia y las migraciones internacionales. Evolución reciente y panorama actual a partir de las cifras. Revista Internacional de Movilidad Humana, 20 (39), 185-210.

Migración Colombia (2012, marzo). Boletín migratorio. Recuperado de http://bit.ly/1Fs3NbD

Migración Colombia (2013, marzo). Boletín migratorio. Recuperado de http://bit.ly/1STT6I0

Migración Colombia (2014, marzo). Boletín migratorio. Recuperado de

http://bit.ly/1 KBqPjL

Migración Colombia (2014, noviembre). Boletín Migratorio. Recuperado de http://bit.ly/1HVHmRq

Migración Colombia (2015, marzo). Boletín migratorio. Recuperado de http://bit.ly/1KBqPjL

Ministerio de Relaciones Exteriores (2014a). Colciencias lanza "Es tiempo de volver", la convocatoria de repatriación de cientificos. Recuperado de http:// bit.ly/1 Nu9POl

Ministerio de Relaciones Exteriores (2014b). Migración Colombia. Recuperado de http://bit.ly/1GEp9pN

Ministerio de Relaciones Exteriores Colombia (2010). La Política Integral Migratoria y el Plan de Retorno Positivo. Recuperado de http://bit.ly/1bH7eD9

Ministerio de Relaciones Exteriores Colombia (2013). Plan Fronteras para la prosperidad. Informe Ejecutivo. Recuperado de http://bit.ly/1HdHKqM

Montoya, D. et al. (2010). Remesas como instrumento de crecimiento económico en Colombia: remesas y el sector de la construcción. Revista de Negocios Internacionales, 3 (2), 64-86.

Muñoz de Bustillo, R. y Antón, J. (2013). La inmigración latinoamericana en España. Contexto, dimensión y características. En Delpino, M. et al. Claves para la comprensión de la inmigración latinoamericana en España. Córdoba: Universidad Católica.

Nagle, L. (2004). Colombian Asylum Seekers: What Practitioners Should Know About the Colombian
Crisis. Georgetown Immigration Law Review, 18, 441-466.

Organización Internacional para las Migraciones (OIM) (2014). Historia. Recuperado de http://www. oim.org.co/historia.html

ONU (2014). Colombianos migran en búsqueda de un mejor futuro. Recuperado de http://bit. ly/19wKV1w

Palma, M. (por publicar). Migración en Colombia: ideas para la gestión de una política pública.

Pardo, D. (2014). "Me voy”. Venezuela se convierte en país de emigrantes. BBC Mundo. Recuperado de http://bbc.in/1tl2XvN

Portes, A. y Rumbaut, R. (2006). Immigrant America: A Portrait. Los Angeles: University of California Press.

Ramírez, C. y Mendoza, L. (2013). Colombia: perfil migratorio 2012. Bogotá: OIM

Ramírez, C., Zuluaga, M. y Perilla, C. (2010). Perfil migratorio de Colombia. Bogotá: OIM.

República de Colombia (2009). Documento Conpes 3603. Política Integral Migratoria. Bogotá: Consejo Nacional de Política Económica y Social.

República de Colombia (2011). Decreto 4062 de 2011 por el cual se crea la Unidad Administrativa Especial Migración Colombia. Bogotá: Departamento Administrativo de la Función Pública.

Revista Semana (2015). El cerebro repatriado. Semana, 1709.

Roa, M. (2010). Usos e inversión de remesas procedentes de España en hogares con experiencia migratoria en Cali. Cali: Centro de Investigaciones CIDSE, Universidad del Valle.

Roll, D. y Gómez, D. (eds.) (2010). Migraciones internacionales. Crisis mundial, nuevas realidades, nuevas perspectivas. Bogotá: IEPRI Universidad Nacional. 
Roll, D. (2012). Iberoamérica soy yo. Bogotá: Editorial Universidad Sergio Arboleda.

Sassen, S. (2005). The Global City: Introducing a Concept. Brown Journal of World Affairs, 9 (2), 27-43.

Solimano, A. (2010). International Migration in the Age of Globalization. Cambridge: University Press.

Stanton, J. (2014). African Asylum Seekers Make Epic Trek Through Latin America To Reach The U.S. Buzzfeed News. Recuperado de http://bzfd. it/1MkcRaQ

Serna, J. (2015). Migrantes, bomba de tiempo que amenaza a Turbo. El Colombiano. Recuperado de http:// bit.ly/1BI3dFd

Suárez, A. (2014). Cada día 116 extranjeros obtienen visa de trabajo en Colombia. El Tiempo.

Tomei, G. (2011). Cultural and Symbolic Dimensions of the migration-development nexus. University of Oxford International Migration Institute, Working Paper Series 2011-30.

UNDOC (2014). Dimensión del delito de tráfico de migrantes en Colombia. Realidades institucionales, legales y judiciales. Bogotá: UNDOC.
Vertovec, S. (1999). Conceiving and Researching Transnationalism. Ethnic and Racial Studies, 22 (2), 447-462.

Vertovec, S. (2007). Circular Migration: The Way Forward in Global Policy? University of Oxford international Migration Institute. Working Papers Series, $2007-4$

Wabgou, M., Vargas, D. y Carabalí, J. (2012). Las migraciones internacionales en Colombia. Investigación y Desarrollo, 20, 1, 142-167.

Wallace, A. (2013). Lo que cruza por el río separa a Colombia y Venezuela. BBCMundo. Recuperado de http://bbc.in/1GEppVZ

Wihtol de Wenden, C. (2013). El fenómeno migratorio en el siglo XXI. México: Fondo de Cultura Económica.

Wimmer, A. y Glick-Schiller, N. (2003). Methodological nationalism, the social sciences and the Study of Migration. International Migration Review, 37 (3), 576-610 